\section{Principais causas básicas da mortalidade infantil no Distrito Federal, Brasil: 1990 a 2000}

\section{Underlying cause of infant mortality in Distrito Federal, Brazil: 1990 to 2000}

Renata Alves Monteiro 1

Bethsáida de Abreu Soares Schmitz 2

1 Área Técnica de Saúde da Criança e Aleitamento Materno. Departamento de Ações Programáticas Estratégicas. Secretaria de Atenção à Saúde. Ministério da Saúde. Esplanada dos Ministérios, Bloco "G", sala 625. Brasília, DF, Brasil. CEP: 70.058-900.

2 Departamento de Nutrição. Faculdade de Ciências da Saúde. Universidade de Brasília. Campus Universitário Darcy Ribeiro. Asa Norte. Brasília, DF, Brasil. CEP: 70.910-900.

\begin{abstract}
Objectives: to characterize the tendency of infant mortality in Distrito Federal (DF) considering underlying causes of death and the avoidable causes criteria between 1990 and 2000 .

Methods: an ecological study using total infant deaths and births. Information systems of the Ministry of Health were used to obtain data. Assessment of underlying death causes was based on the International Classification of Disease. For avoidable causes analysis related to the criteria established by the SEADE foundation was used.

Results: deaths from perinatal problems were predominant, followed by deaths related to congenital anomalies. However, greater reduction occurred in the mortality rate resulting from parasitic and infection diseases. When each coefficient was analyzed separately, neonatal deaths, avoidable deaths through early diagnosis and treatment and non-avoidable deaths are outstanding

Conclusions: the need for effective interventions in the causes of death and child mortality, specially related with the care during pregnancy, delivery and after-birth in addition to intervention by civil society sectors was determined.
\end{abstract}

Key words Infant mortality, Postneonatal mortality; Underlying cause of death

\section{Resumo}

Objetivos: caracterizar a tendência da mortalidade infantil no Distrito Federal por causa básica de morte e segundo causas evitáveis entre 1990 e 2000.

Métodos: estudo ecológico a partir do total de óbitos infantis e nascimentos ocorridos, utilizando-se os sistemas de informação produzidos pelo Ministério da Saúde. Para avaliação, segundo causa básica de morte, usou-se a Classificação Internacional de Doenças. Para a análise, segundo causas evitáveis, foi utilizado o critério estabelecido pela Fundação SEADE.

Resultados: predominaram os óbitos devido a afecções perinatais, seguidos das mortes em decorrência de anomalias congênitas, porém a maior redução ocorreu no coeficiente de mortalidade por doenças infecciosas e parasitárias. Quando se avalia cada coeficiente em separado, destacam-se, nos óbitos neonatais, aqueles redutíveis por meio de ações de diagnóstico e tratamento precoces e os não evitáveis. Entre as mortes pós-neonatais, destacam-se aquelas também redutíveis por ações precoces de diagnóstico e tratamento e por meio de parcerias com outros setores, como aqueles óbitos por diarréia e infecções respiratórias agudas.

Conclusões: verifica-se a necessidade de intervenção efetiva nas causas de morte e componentes da mortalidade infantil, principalmente naquelas relacionadas ao cuidado durante a gravidez, parto $e$ puerpério, além daquelas que implicam na ação conjunta de vários setores.

Palavras-chave Mortalidade infantil, Mortalidade pós-neonatal, Causa básica de morte 


\section{Introdução}

O conhecimento dos níveis de mortalidade infantil de uma dada comunidade constitui um elemento precioso para a análise da situação de saúde, auxiliando na avaliação de programas e na vigilância epidemiológica dos agravos à saúde. Também orienta na identificação de grupos populacionais mais expostos ao risco de adoecer e morrer. ${ }^{1}$

A probabilidade de falecer no primeiro ano de vida possui uma distribuição desigual no território brasileiro, mesmo em localidades onde o coeficiente de mortalidade infantil (CMI) já atingiu valores abaixo de $20 \%$ o nascidos vivos. Isso pode ser verificado quando observadas as causas básicas dos óbitos ocorridos no período neonatal e pós-neonatal. ${ }^{2}$

As causas da mortalidade neonatal estão normalmente relacionadas a problemas congênitos, a fatores da saúde materna e a complicações durante a gestação e parto. No Brasil, ao contrário do que ocorre em países desenvolvidos, as complicações que se estabelecem durante a gestação e parto são as que representam as maiores causas de morte entre os neonatos, sendo responsáveis por, aproximadamente, $80 \%$ das mortes neonatais e $50 \%$ da mortalidade infantil. 3

Entre as causas da mortalidade pós-neonatal estão, predominantemente, aquelas relacionadas aos riscos ambientais, como as infecções respiratórias agudas e as doenças infecciosas intestinais, além das deficiências nutricionais. No Brasil, observa-se que há uma discrepância importante entre as várias regiões no que diz respeito às causas de óbito neste componente, principalmente por estarem diretamente relacionadas às condições socioeconômicas e de vida, podendo representar até $18 \%$ da mortalidade infantil total. 3

Apesar de os óbitos por doenças infecto-parasitárias terem diminuído nos últimos anos, doenças como a diarréia, as septicemias e as broncopneumonias ainda levam a óbito muitas crianças brasileiras com menos de um ano de idade, revelando condições de vida adversas em distintos segmentos da população. 4

A mortalidade infantil no Distrito Federal, DF, como em diversos estados do país, vem sofrendo uma redução gradativa nas últimas décadas, passando de 41, em 1980, para 23,8\% nascidos vivos, em 1989. Nesse mesmo período, no DF, ocorreu uma predominância de óbitos em decorrência das afecções perinatais, seguida de óbitos por anomalias congênitas e doenças infecciosas e parasitárias. ${ }^{5}$ Esses dados são diferentes da situação encontrada em algumas regiões menos favorecidas do país, nas quais, na década de 80 , se mantinham, ainda, proporções elevadas de óbitos por doenças infectoparasitárias e doenças do aparelho respiratório, além dos óbitos por afecções perinatais. 4

O objetivo do presente trabalho é caracterizar, no período de 1990 a 2000, o comportamento da mortalidade infantil no Distrito Federal por causa básica de morte e segundo a classificação de causas evitáveis. Visa a subsidiar ações de planejamento em saúde pública, com o intuito de atuação específica que leve a um maior impacto na redução deste coeficiente, principalmente nas causas de morte mais expressivas.

\section{Métodos}

A população estudada consistiu do total de óbitos de menores de um ano e de nascimentos de crianças registrados, residentes no Distrito Federal, no período de 1990 a 2000. Foi realizado um estudo ecológico, utilizando-se informações oriundas de Sistemas de Informação do Ministério da Saúde sobre mortalidade (SIM) e sobre nascidos vivos (SINASC), específicas do Distrito Federal.

O Distrito Federal, onde se localiza a capital brasileira, ocupa uma área de 5.789,16 Km² e está dividido atualmente em 19 Regiões Administrativas (RAs). 6

De acordo com o censo 2000, o DF possui uma população de 2.051 .000 habitantes, dos quais, aproximadamente, $2,0 \%$ são menores de um ano. ${ }^{7}$

Em 1998, o DF era o primeiro colocado entre todos os estados brasileiros, juntamente com o Rio Grande do Sul, em relação ao Índice de Desenvolvimento Humano (IDH). O IDH, preconizado pela Organização das Nações Unidas, é uma medidaresumo do desenvolvimento humano com base em renda, educação e expectativa de vida. ${ }^{8}$

O IDH do DF foi de 0,869 em 1998. Esse valor é considerado elevado, indicando desenvolvimento humano alto, sendo superior ao IDH do país em $2000(0,757)$, que foi o $73^{\circ}$ colocado na relação de IDH no mundo. $\mathrm{O}$ valor de IDH apresentado pelo DF em 1998 é comparável ao de países como Barbados e Brunei, que são o $32^{\circ}$ e $33^{\circ}$ colocados no IDH mundial. 8

Os dados referentes aos óbitos infantis foram obtidos junto ao Sistema de Informação sobre Mortalidade (SIM), sistema coordenado pelo Ministério da Saúde. Os dados relativos ao DF são encaminhados ao SIM pelo Departamento de Saúde Pública da Secretaria de Saúde do Distrito Federal. ${ }^{9}$

As informações sobre os nascidos vivos no 
Distrito Federal referentes aos anos de 1990 a 1993 foram obtidas junto ao sistema de informação da própria Secretaria de Saúde do Distrito Federal. A partir de 1994, os dados tiveram como fonte o Sistema de Informação sobre Nascidos Vivos (SINASC). Tanto o sistema SIM quanto o SINASC são atualmente disponibilizados pela Secretaria de Vigilância à Saúde do Ministério da Saúde na forma de cd-rom e em um site produzidos pelo Banco de Dados do Sistema Único de Saúde (DATASUS).3,9

A causa básica de morte é definida como ... a doença ou lesão que iniciou a cadeia de acontecimentos patológicos que conduziram diretamente à morte, ou as circunstâncias do acidente ou violência que produziram a lesão fatal. (Ministério da Saúde; 1991: 38)1

A causa básica foi codificada no SIM de acordo com a Classificação Internacional de Doenças (CID), regulamentada pela Organização Mundial de Saúde (OMS) e adotada pelo Ministério da Saúde. Foram utilizadas, visando a análise, a nona revisão da Classificação Internacional de Doenças, até o ano de 1995 , e a $10^{\text {a }}$ revisão, a partir de $1996 .{ }^{9}$ A análise por causa básica de óbito foi expressa por capítulos da CID e segundo os critérios de evitabilidade.

A codificação em causas evitáveis foi realizada, conforme critério da Fundação Sistema Estadual de Análise de Dados (SEADE), adotado pelo Ministério da Saúde. 3

A tendência do coeficiente de mortalidade infantil por causa e da mortalidade infantil proporcional por causa entre 1990 e 2000 foi verificada por meio do modelo de regressão linear. Tal modelo tem alto poder do ponto de vista estatístico, além de ser de fácil interpretação e se adaptar aos dados apresentados. 10

A equação que representa o modelo de regressão linear é $\mathrm{Y}=\beta_{0}+\beta_{1} \mathrm{X}$, onde os valores de $\mathrm{Y}$ e $\mathrm{X}$ são as variáveis dependente e independente, respectivamente, e $\beta_{0}$ e $\beta_{1}$ são os parâmetros da reta que melhor se ajustam aos dados. $O$ valor de $\beta_{0}$ indica $o$ valor da interceptação no eixo de $\mathrm{Y}$ e $\beta_{1}$ é o incremento médio anual. Foi analisado, também, o coeficiente de determinação $\left(\mathrm{R}^{2}\right)$, sendo considerado como nível de significância a inferior a 5\%.

Os dados foram processados e analisados em microcomputador, utilizando-se o programa de tabulação Tabwin, versão 3.2 e o programa Epi-info 6.04. Foi utilizado o programa Excel for Windows (versão 5.02) no cálculo dos coeficientes e proporções de óbitos por causa básica e causa evitável, bem como na elaboração do modelo de regressão linear.

\section{Resultados}

Houve uma variação percentual de $-45,2 \%$ no CMI no Distrito Federal entre 1990 e 2000, passando de 26,3 para $14,4 \%$ onascidos vivos. O percentual médio de decréscimo anual foi de $5,86 \%$. Houve redução no coeficiente de mortalidade em todas as principais causas básicas de morte na faixa etária estudada no período, como se pode observar na Tabela 1.

Quando avaliada a mortalidade por causa básica de morte, verifica-se que há uma maior ocorrência no DF de óbitos por afecções perinatais em todo o intervalo de tempo estudado. Quando somadas aos óbitos por anomalias congênitas, as mortes por afecções perinatais passaram a representar, após 1995, mais de $70 \%$ dos óbitos em menores de um ano (Figura 1).

A taxa de mortalidade por afecções perinatais diminuiu $38,8 \%$ ( $p<0,05)$, passando de 12,9 a $7,9 \%$ o nascidos vivos (Tabela 1). Porém, a proporção de óbitos por essa causa no total de óbitos em menores de um ano aumentou, provavelmente devido ao decréscimo de mortes por outras causas, principalmente por doenças infecto-parasitárias. (Figura 1) As mortes por essa última causa sofreram um decréscimo de $84,2 \%(p<0,05)$ em seu coeficiente entre 1990 e 2000. A redução na proporção dos óbitos por doenças infecto-parasitárias no total de óbitos ocorridos no primeiro ano de vida foi de $72,8 \%$, passando a representar, em 2000 , a quarta principal causa de morte entre as crianças menores de um ano.

A mortalidade por doenças do aparelho respiratório e por deficiências nutricionais também sofreu redução no período estudado, representando a segunda e terceira maiores reduções no coeficiente de mortalidade por causa básica e na proporção de óbitos por causa básica no período.

Quando analisados os óbitos segundo causas evitáveis, observa-se que ocorreu diminuição no coeficiente e na proporção de causas redutíveis e aumento na proporção de causas não-evitáveis. Esse último item passou de 4,0\%, em 1990, para, aproximadamente, $24,0 \%$, em 2000. (Tabela 2)

No período estudado, como está destacado na Tabela 2, houve diminuição na proporção de óbitos redutíveis por prevenção, diagnóstico e tratamento precoces, apesar de estes, em 2000, representarem, ainda, as causas responsáveis pela maior proporção dos óbitos $(35,5 \%)$. 
Coeficiente de mortalidade infantil, segundo principais causas de morte e análise da regressão pelo modelo linear. Distrito Federal (DF), Brasil, 1990 a 2000.

\begin{tabular}{lcccc}
\hline Causa Básica de Morte & $\mathbf{1 9 9 0}$ & $\mathbf{2 0 0 0}$ & $\boldsymbol{\Delta} \%$ & Valor de $\boldsymbol{p}$ \\
\hline Algumas afecções perinatais & 12,9 & 7,9 & $-38,8$ & 0,9575 \\
Doenças infecciosas e parasitárias & 3,8 & 0,6 & $-84,2$ & 0,6215 \\
Anomalias congênitas & 3,5 & 3,3 & $-5,7$ & 0,0006 \\
Doenças do aparelho respiratório & 3,0 & 0,7 & $-76,7$ & 0,8516 \\
Doenças endócrinas e nutricionais & 0,9 & 0,3 & $-66,7$ & 0,8279 \\
Doenças do sistema nervoso & 0,6 & 0,4 & $-33,3$ & 0,4959 \\
Demais causas & 1,7 & 1,3 & $-23,5$ & 0,4623 \\
Total & 26,3 & 14,4 & $-45,2$ & 0,001 \\
\hline
\end{tabular}

$\mathrm{Y}=$ Coeficiente de mortalidade (\%o nascidos vivos); $\mathrm{X}=$ ano; ${ }^{\mathrm{R}}{ }^{2}=$ Coeficiente de determinação;

\section{Figura 1}

Mortalidade infantil proporcional, segundo principais causas básicas de morte. Distrito Federal (DF), Brasil, 1990 a 2000.

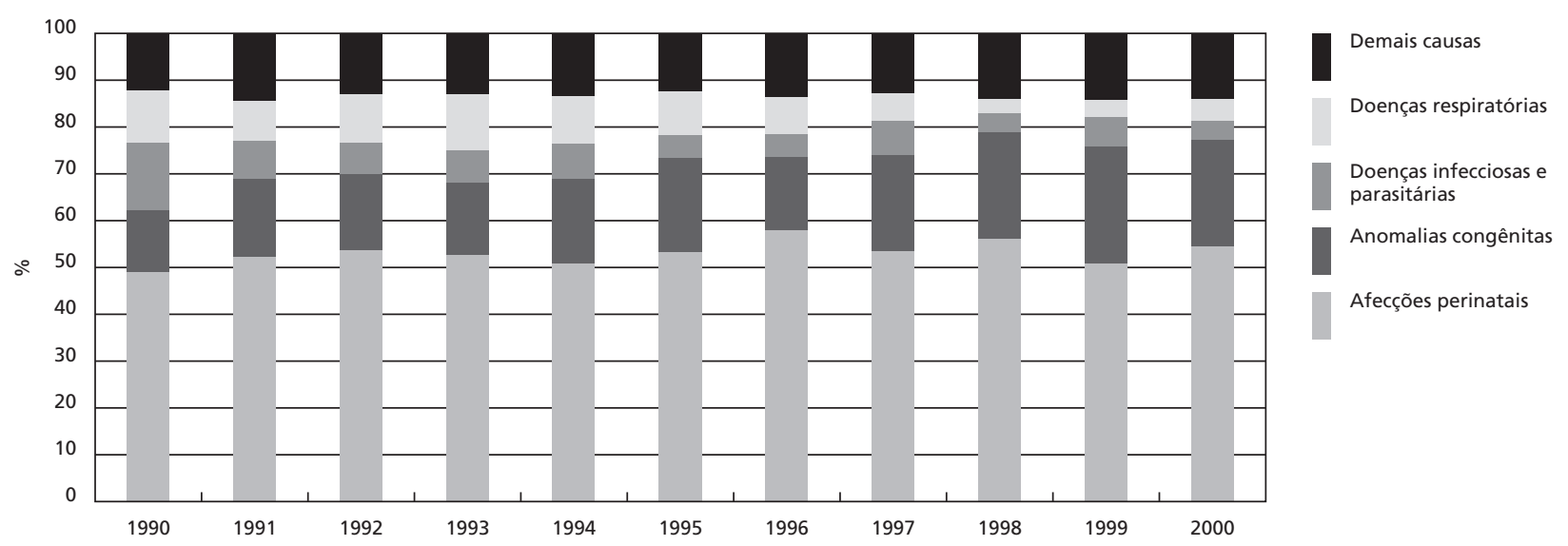

Não foi estabelecida variação percentual significante $(-5,8 \%, p>0,05)$ na proporção dos óbitos redutíveis por adequado controle na gravidez, apesar de ter havido redução de 50,0\% no coeficiente de mortalidade por essa causa $(p<0,05)$. Deve-se destacar também as causas redutíveis por meio de parcerias com outros setores, pois obtiveram o menor percentual de redução do coeficiente entre 1990 e 2000. Além disso, sofreram um aumento significante $(30,4 \%, p<0,05)$ de sua representatividade na mortalidade infantil. (Tabela 2)

Quando se analisa, em separado, cada componente da mortalidade infantil, observa-se, no Distrito Federal, um predomínio de óbitos no período neonatal. Em decorrência disso, fatores ligados à gestação e ao parto tiveram influência especial sobre a redução dos óbitos neonatais, como se pode observar na Tabela 3. 
Coeficiente de mortalidade infantil (CMI) e mortalidade infantil proporcional (MIP), segundo causas evitáveis. Distrito Federal (DF), Brasil, 1990 a 2000.

\begin{tabular}{|c|c|c|c|c|c|c|c|c|}
\hline \multirow{2}{*}{ Critério de causas evitáveis } & \multicolumn{4}{|c|}{ CMI } & \multicolumn{4}{|c|}{ MIP } \\
\hline & 1990 & 2000 & $\Delta \%$ & Valor de $p$ & 1990 & 2000 & $\Delta \%$ & Valor de $p$ \\
\hline Redutíveis por imunoprevenção & 0,4 & 0 & -100 & - & 1,6 & 0 & -100 & - \\
\hline $\begin{array}{l}\text { Redutíveis por adequado } \\
\text { controle na gravidez }\end{array}$ & 1,8 & 0,9 & $-50,0$ & $<0,001$ & 6,9 & 6,5 & $-5,8$ & 0,77 \\
\hline $\begin{array}{l}\text { Redutíveis por adequada } \\
\text { atenção ao parto }\end{array}$ & 2,5 & 1,1 & $-56,0$ & $<0,001$ & 9,4 & 7,4 & $-21,3$ & 0,15 \\
\hline $\begin{array}{l}\text { Redutíveis por ações prevenção, } \\
p<0,000001 \\
\text { diagnóstico e tratamento precoces }\end{array}$ & 12,5 & 5,1 & $-59,2$ & $<0,001$ & 47,6 & 35,5 & $-25,4$ & \\
\hline $\begin{array}{l}\text { Redutíveis através de parcerias } \\
\text { com outros setores }\end{array}$ & 4,5 & 3,2 & $-28,9$ & 0,002 & 17,1 & 22,3 & 30,4 & 0,008 \\
\hline $\begin{array}{l}\text { Não evitáveis } \\
p<0,000001\end{array}$ & 1,0 & 3,4 & 226,9 & $<0,001$ & 4,0 & 23,9 & 497,5 & \\
\hline Mal definidas & 0,4 & 0,6 & 50,0 & 0,07 & 1,4 & 4,5 & 221,4 & $<0,001$ \\
\hline Não classificadas & 3,2 & 0,0 & $-100,0$ & - & 12,2 & 0,0 & $-100,0$ & - \\
\hline
\end{tabular}

Tabela 3

Coeficiente de mortalidade neonatal (CMN) e mortalidade neonatal proporcional (MNP), segundo causas evitáveis. Distrito Federal, Brasil,1990 a 2000.

\begin{tabular}{|c|c|c|c|c|c|c|c|c|}
\hline \multirow{2}{*}{ Critério de causas evitáveis } & \multicolumn{4}{|c|}{ CMI } & \multicolumn{4}{|c|}{ MIP } \\
\hline & 1990 & 2000 & $\Delta \%$ & Valor de $p$ & 1990 & 2000 & $\Delta \%$ & Valor de $p$ \\
\hline Redutíveis por imunoprevenção & 0,0 & 0,0 & - & - & 0,1 & 0 & -100 & - \\
\hline Redutíveis por adequado controle na gravidez & 1,7 & 0,8 & $-52,9$ & $<0,001$ & 6,4 & 5,4 & $-15,6$ & 0,4 \\
\hline Redutíveis por adequada atenção ao parto & 2,3 & 1,0 & $-56,5$ & $<0,001$ & 8,6 & 7,1 & $-17,4$ & 0,25 \\
\hline $\begin{array}{l}\text { Redutíveis por ações prevenção, } \\
\text { diagnóstico e tratamento precoces }\end{array}$ & 7,2 & 3,7 & $-48,6$ & $<0,001$ & 27,5 & 25,5 & $-7,3$ & 0,36 \\
\hline $\begin{array}{l}\text { Redutíveis através de parcerias } \\
\text { com outros setores }\end{array}$ & 0,5 & 1,3 & 160,0 & $<0,001$ & 1,9 & 9,1 & 378,9 & $<0,001$ \\
\hline Não evitáveis & 0,7 & 2,9 & 314,3 & $<0,001$ & 2,7 & 20,3 & 651,9 & $<0,001$ \\
\hline Mal definidas & 0,1 & 0,3 & 200,0 & 0,08 & 0,3 & 1,9 & 533,3 & $<0,001$ \\
\hline Não classificadas & 2,9 & 0,0 & $-100,0$ & - & 11,1 & 0,0 & $-100,0$ & - \\
\hline Total & 15,4 & 10,0 & $-35,1$ & $<0,001$ & 58,6 & 69,2 & 18,1 & 0,001 \\
\hline
\end{tabular}

$* p<0,000001$ 
Entre 1990 e 2000, o coeficiente de mortalidade neonatal teve variação percentual de $-35,1 \%$, passando de 15,4 para 10,0\%o nascidos vivos. As categorias de causas redutíveis responsáveis por essa diminuição foram as relacionadas à atenção ao parto, controle na gravidez e ações de prevenção, diagnóstico e tratamento precoces. Apesar disto, esse último grupo de causas redutíveis foi responsável, em 2000, pela maior proporção dos óbitos ocorridos em menores de um ano no DF $(25,5 \%)$, vindo, a seguir, as causas não evitáveis $(20,3 \%)$.

Na Tabela 4, observa-se que, entre os óbitos de menores de um ano, a maior redução no coeficiente de mortalidade se deu no componente pós-neonatal, que passou de 10,5 para $4,3 \%$ nascidos vivos, representando uma variação de 59,0\% $(p<0,05)$. A maior contribuição para essa variação foi dada pelas causas redutíveis por prevenção, diagnóstico e tratamento precoces $(-73,6 \%, p<0,05)$ e por causas redutíveis por meio da parceria com outros setores, cujas ações têm influência, por exemplo, na diarréia e nas doenças respiratórias. Manteve-se baixo, durante todo o intervalo de tempo estudado, o coeficiente de mortalidade por causas não evitáveis no período pósneonatal.

Tabela 4

Coeficiente de mortalidade pós-neonatal (CMPN) e mortalidade pós-neonatal proporcional (MPNP), segundo causas evitáveis. Distrito Federal (DF), Brasil, 1990 a 2000.

\begin{tabular}{|c|c|c|c|c|c|c|c|c|}
\hline \multirow{2}{*}{ Critério de causas evitáveis } & \multicolumn{4}{|c|}{ CMPN } & \multicolumn{4}{|c|}{ MPNP } \\
\hline & 1990 & 2000 & $\Delta \%$ & Valor de $p$ & 1990 & 2000 & $\Delta \%$ & Valor de $p$ \\
\hline Redutíveis por imunoprevenção & 0,4 & 0 & -100 & - & 1,5 & 0 & -100 & - \\
\hline $\begin{array}{l}\text { Redutíveis por adequado controle } \\
\text { na gravidez }\end{array}$ & 0,0 & 0,1 & $-0,1$ & 0,28 & 0,1 & 0,6 & 600,0 & 0,1 \\
\hline $\begin{array}{l}\text { Redutíveis por adequada atenção } \\
\text { ao parto }\end{array}$ & 0,1 & 0,0 & $-100,0$ & 0,4 & 0,2 & 0,1 & $-50,0$ & 0,6 \\
\hline $\begin{array}{l}\text { Redutíveis por ações prevenção, diagnóstico } \\
\text { e tratamento precoces }\end{array}$ & 5,3 & 1,4 & $-73,6$ & $<0,001$ & 20,0 & 9,8 & $-51,0$ & $<0,001$ \\
\hline $\begin{array}{l}\text { Redutíveis através de parcerias } \\
\text { com outros setores }\end{array}$ & 4,0 & 1,9 & $-52,5$ & $<0,001$ & 15,2 & 13,2 & $-13,2$ & 0,24 \\
\hline Não evitáveis & 0,3 & 0,5 & 66,7 & 0,19 & 1,3 & 3,6 & 176,9 & $<0,001$ \\
\hline Mal definidas & 0,2 & 0,4 & 100,0 & 0,13 & 0,7 & 2,6 & 271,4 & 0,002 \\
\hline Não classificadas & 0,3 & 0,0 & $-100,0$ & - & 1,0 & 0,0 & $-100,0$ & - \\
\hline Total & 10,5 & 4,3 & $-59,0$ & $<0,001$ & 40,0 & 30,0 & $-25,0$ & $<0,001$ \\
\hline
\end{tabular}

\section{Discussão}

Mesmo em países desenvolvidos, que já atingiram níveis muito baixos de mortalidade infantil, essa é tratada como problema prioritário de saúde, principalmente devido à probabilidade de atuação no seu controle com ações específicas em saúde e educação. ${ }^{11}$ Além disso, características epidemiológicas levam a mortalidade infantil a ser mais encarada como um evento "sentinela" de uma ocorrência potencialmente evitável do que apenas um indicador de risco agregado. 12

O uso de causas básicas de morte é de grande importância no estudo da mortalidade, pois é intervindo sobre essas que, quase sempre, as ações poderão ser mais eficientes e eficazes sobre a redução da mortalidade. A prevenção da afecção inicial diminui, com certeza, um grande número de 
mortes prematuras e evitáveis, além de reduzir os custos sociais e econômicos decorrentes de patologias e problemas de saúde. 1

No entanto, o uso de causas básicas pode subestimar a contribuição de outros problemas como, por exemplo, das deficiências nutricionais que nem sempre aparecem como causa básica, mas sim como causa associada. A análise por causas múltiplas poderia auxiliar na determinação da importância relativa das várias causas para a ocorrência de um óbito. 5

Apesar de a utilização da causa de morte auxiliar no entendimento do comportamento da mortalidade infantil, contribuindo assim para a avaliação dos serviços de saúde e condições de vida de uma dada população, imprecisões na declaração dessa variável podem comprometer a consistência do indicador utilizado, independente da boa cobertura do Sistema de Informação. ${ }^{2}$ No Distrito Federal, na década anterior, observou-se que a informação relativa a causa básica de morte estava presente em $100,0 \%$ das declarações de óbito, indicando, pelo menos em relação ao grau de preenchimento, uma condição de adequação para realização de estudos com essa variável.13

Porém, apesar dos dados do DF serem considerados de boa qualidade, 14 há de se destacar a carência de pesquisas que avaliem a qualidade dos dados relacionada ao adequado preenchimento da informação referente à causa básica de morte no DF. Além disso, se faz importante investir na sensibilização dos profissionais responsáveis pelo preenchimento da DO uma vez que estudos em outras localidades indicaram a necessidade de melhoria da informação dessa variável.15,16

O DF apresenta, atualmente, um dos menores coeficientes de mortalidade infantil do país, 2 havendo uma predominância, cada vez maior, do componente neonatal, o que se reflete no perfil das causas básicas de morte, principalmente no que tange ao critério de evitabilidade. Esse comportamento já era observado a partir da metade da década anterior no Distrito Federal, 5 e em outros locais do país, como na Região Sudeste e Região Sul.17,18

Quando há predomínio de mortes infantis no período pós-neonatal, isso indica grande influência de fatores externos, como os relacionados às doenças infecciosas e às deficiências nutricionais. ${ }^{19}$ Porém, nos últimos anos, as causas relacionadas ao período neonatal têm apresentado significância cada vez maior, seguindo o padrão já existente nos países desenvolvidos e em alguns países em desenvolvimento, como em Cuba, Chile e Porto Rico. ${ }^{20-22}$ Isso ocorre devido a um maior controle dos fatores que se relacionam à mortalidade pós-neonatal, que têm, portanto, maior expressão sobre algumas causas de morte, como as doenças infecto-parasitárias. Essas, no DF, passaram do segundo lugar, em 1990, para o quarto lugar, em 2000, como causa básica de óbitos em menores de um ano. O mesmo comportamento também foi o observado nos dados gerais do Brasil, influenciado principalmente pelas regiões Sudeste e Sul. 17

Quando correlacionados fatores que pudessem estar influenciando a redução da mortalidade pósneonatal, observa-se que, no período estudado, apesar de não haver ocorrido ampliação no número de leitos oferecidos à população no DF, bem como no número de centros de saúde e do percentual de famílias com abastecimento adequado de água, 3,6 houve ampliação do Programa Saúde da Família e dos Agentes Comunitários de Saúde,23,24 responsáveis por ações básicas de prevenção e promoção da saúde. Além disso, deve-se destacar que a cobertura quanto à imunização é alta, superando, em algumas localidades, o esperado em mais de $100 \%, 24 \mathrm{o}$ que pode ser evidenciado pela ausência, em 2000, de óbitos redutíveis por imunoprevenção, indicando a eficiência das campanhas de imunização ocorridas no Distrito Federal, no período de 1990 a 2000. Ocorreu também, no DF, aumento na prevalência de aleitamento materno e na mediana de aleitamento materno exclusivo, principalmente nas cidades de menor renda familiar. 25 Essas condições influenciam diretamente na redução da morbimortalidade principalmente por doenças prevalentes no período pósneonatal.

A proporção de óbitos não evitáveis, principalmente no período neonatal, é superior à encontrada em outros estudos e sofreu aumento expressivo desde 1980, cuja proporção desse grupo de causas era de apenas 1,7\%.3,12,18 Apesar de o maior número de óbitos por causas não evitáveis indicarem que algumas mudanças sociais e estratégias de saúde têm surtido efeito sobre os óbitos pós-neonatais, esse fato não consegue mascarar mortes que poderiam ser evitadas por ações simplificadas dos serviços de saúde, e por modificações estruturais nas condições de vida da população. Isso pode ser observado pelos coeficientes ainda altos de óbitos por causas redutíveis por ações de prevenção, diagnóstico e tratamento precoces e por meio de parceria com outros setores, indicando necessidade de melhoria e ampliação das ações no setor saúde que podem agir sobre esse grupo de causas.

Apesar da queda na proporção de mortes redutíveis por adequada atenção ao parto, revelando melhoria da assistência imediata após o mesmo, grande parte dos óbitos poderia ser evitada se fossem 
ampliados o oferecimento e a adequação do atendimento pré-natal em relação ao número de consultas e a sua qualidade, assim como da assistência ao recém-nascido. 20 Isso indica a necessidade de maior investimento na prevenção e na redução dos óbitos por causas relacionadas com afecções e complicações na saúde materna, com destaque à melhoria do estado nutricional materno.

O aumento na proporção de óbitos por causas mal definidas $(p=0,001)$ indica a necessidade de aperfeiçoamento do sistema de informação sobre mortalidade e de vigilância sobre os aspectos relacionados ao diagnóstico da causa básica de morte e a importância do preenchimento adequado dessa informação.

\section{Referências}

1. Ministério da Saúde. FUNASA (Fundação Nacional de Saúde), Coordenação de Informações Epidemiológicas. Análise de mortalidade: delineamentos básicos. Brasília: Distrito Federal (DF): O Ministério; 1991.

2. Rede Interagencial de Informações para a Saúde. Indicadores básicos para a saúde no Brasil: conceitos e aplicações. Brasília (DF): Organização Pan-Americana de Saúde; 2002.

3. Ministério da Saúde. Secretaria de Vigilância à Saúde. Coordenação de Informações Epidemiológicas. Sistema de Informações sobre Mortalidade. Brasília (DF): O Ministério; Disponível em: <http://www.datasus. gov.br>. [2003 jan 8].

4. Carvalho BG, Costa MCN. Mortalidade infantil e seus componentes em Salvador-BA, 1980-1991. Inf Epidemi ol Sist Único de Saúde 1998; 7: 35-41.

5. Schmitz BAS, Bezerra VLVA, Turnes O. Mortalidade infantil no Distrito Federal segundo sexo e causa básica de morte: 1980-1989. Rev Saúde Distrito Federal 2000; 11 14-21.

6. Anuário estatístico do Distrito Federal, 2000 [cd-rom]. Brasília (DF): Secretaria do Estado de Desenvolvimento Urbano e Habitação; 2002.

7. IBGE (Instituto Brasileiro de Geografia e Estatística). Base de informações por setor censitário: censo demográfico 2000: resultados do universo. São Paulo: O Instituto; 2002.

8. PNUD (Programa das Nações Unidas para o Desenvolvimento). Relatório do desenvolvimento humano,:2002. Lisboa: Mensagem; 2002.

9. Ministério da Saúde. Secretaria de Saúde. Departamento de Saúde. Atividades e funções do sistema de informação DF (SIS). Brasília (DF): O Ministério; 2001.

10. Latorre MRDO, Cardoso MRA. Análise de séries temporais em epidemiologia: uma introdução sobre os aspectos
Somente por meio de ações integradas e políticas públicas específicas voltadas para ambos os componentes da mortalidade infantil será possível aumentar a velocidade de queda dos índices de mortalidade infantil nos diversos estados brasileiros e no Distrito Federal, revertendo uma situação onde crianças com potenciais probabilidades de sobrevivência vão a óbito, não só por condições inadequadas de assistência em saúde durante a gestação e após o nascimento, mas também por todo um ambiente sócioeconômico que se traduz em condições de vida prejudiciais à saúde. metodológicos. Rev Bras Epidemiol 2001; 4: 145-52.

11. Leal MC, Szwarcwald CL. Evolução da mortalidade neonatal no Estado do Rio de Janeiro, Brasil, de 1979 a 1993. 1 - Análise por grupo etário segundo região de residência. Rev Saúde Pública 1996; 30: 403-12.

12. Campos TP, Carvalho MS, Barcellos, CC. Mortalidade infantil no Rio de Janeiro, Brasil: áreas de risco e trajetória dos pacientes até os serviços de saúde. Rev Panam Salud Pública 2000; 8: 164-71.

13. Schmitz BAS, Bezerra VLVA, Turnes O. Análise do preenchimento de declarações de óbito de menores de cinco anos no Distrito Federal (Brasil): 1980-1989. Rev Saúde Distrito Federal 2000; 11: 31-6.

14. Pereira MG. Epidemiologia: teoria e prática. Rio de Janeiro: Guanabara Koogan; 1997.

15. Nobre LC, Victora CG, Barros FC, Lombardi C, Teixeira AMB, Fuchs SC. Avaliação da qualidade da informação sobre a causa básica de óbitos infantis no Rio Grande do Sul (Brasil). Rev Saúde Pública 1989; 23: 207-13.

16. Niobey FML, Cascão AM, Duchiade MP, Sabroza PC. Qualidade do preenchimento de atestados de óbito de menores de um ano na Região Metropolitana do Rio de Janeiro. Rev Saúde Pública 1990; 24: 311-8.

17. Mello-Jorge MHP, Gotlieb SLD, Laurenti R. A saúde no Brasil: análise do período 1996 a 1999. Brasília (DF): Organização Pan-Americana de Saúde; 2001.

18. Gomes JO, Santo AH. Mortalidade infantil em município da região Centro-Oeste Paulista, Brasil, 1990 a 1992. Rev Saúde Pública 1997; 31: 330-41.

19. Taucher E, Jofré I. Mortalidad infantil en Chile: el gran descenso. Rev Med Chile 1997; 125: 1225-35.

20. Simões CCS. Perfis de saúde e de mortalidade no Brasil: uma análise de seus condicionantes em grupos populacionais específicos. Brasília (DF): Organização PanAmericana de Saúde; 2002. 
21. Szot J, Moreno C. La salud de la madre y el niño en el Servicio de Salud Metropolitano Sur Oriente y en Chile: una visión del periodo 1980-1998. Rev Chil Obstet Ginecol 2001; 66: 119-23.

22. OPS (Organización Panamericana de la Salud). Estadísticas de salud de las Américas: edición 1998. Washington (DC): La Organización; 1998. (Publicación científica, 567).

23. Ministério da Saúde. Secretária Executiva. Programa Saúde da Família - PSF). Brasília (DF): O Ministério; 2001.

24. Ministério da Saúde. Secretaria Executiva. Programa Agentes Comunitários de Saúde - PACS. Brasília (DF): O Ministério; 2000.

25. Sena MCF. O aleitamento materno no Distrito Federal nos anos 90. Brasília: Fundação de Ensino e Pesquisa em Ciências da Saúde; 2002.

Recebido em 14 de janeiro de 2004

Versão final apresentada em 14 de julho de 2004

Aprovado em 26 de outubro de 2004 